\title{
Urinary steroid profile after the completion of concentric and concentric/eccentric trials with the same total workload
}

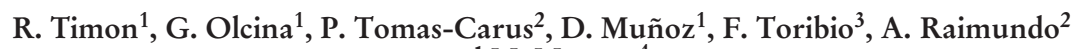 \\ and M. Maynar ${ }^{4}$ \\ ${ }^{1}$ Department of Physical Education and Sport. Sport Science Faculty, University of \\ Extremadura, Cáceres, Spain, ${ }^{2}$ Department of Sport and Health, University of Evora, \\ Evora, Portugal, ${ }^{3}$ Department of Analytical Chemistry and Electrochemistry, School of \\ Sciences, University of Extremadura, Badajoz, Spain, ${ }^{4}$ Department of Physiology, Sport \\ Science Faculty, University of Extremadura, Cáceres, Spain
}

(Received on December, 2008)

R. TIMON, G. OLCINA, P. TOMAS-CARUS, D. MUÑOZ, F. TORIBIO, A. RAIMUNDO and M. MAYNAR. Urinary steroid profile after the completion of concentric and concentric/eccentric trials with the same total workload. J Physiol Biochem, 65 (2), 105-112, 2009.

High intensity strength training causes changes in steroid hormone concentrations. This could be altered by the muscular contraction type: eccentric or concentric. The aim of this study was to compare the effect of the completion of a short concentric $(\mathrm{CON})$ and concentric/eccentric $(\mathrm{CON} / \mathrm{ECC})$ trial on the urinary steroid profile, both with the same total work. 18 males performed the trials on an isokinetic dynamometer (BIODEX III) exercising quadriceps muscles, right and left, on different days. Trial 1(CON): 4x10 Concentric knee extension + relax knee flexion, speed $60 \%$ second; rest 90 seconds between each series and 4 minutes between each leg exercise. Trial 2(CON/ECC): 4x5 concentric knee extension + Eccentric knee flexion under similar conditions. Urine samples were taken before the exercise and one hour after finishing it. Androsterone, Etiocholanolone, DHEA, Androstenedione, Testosterone, Epitestosterone, Dehydrotestosterone, Estrone, B-Estradiol, Tetrahydrocortisone, Tetrahydrocortisol, Cortisone and Cortisol (free, glucoconjugated and sulfoconjugated) urinary values were determined using gas chromatography/mass spectrometry techniques. No significant differences were noted in Total Work and Average Peak Torque, although Maximum Peak Torque in the CON/ECC trial was higher than in the CON trial. These results demonstrate no changes in the steroid profile before and after trials, or when comparing CON to CON/ECC trials. The data suggest that eccentric contractions do not cause hormonal changes different to the ones produced by concentric contractions, when they are performed in strength short trials with the same total workload.

Key words: Androgens, Corticosteroids, Workload, Concentric, Eccentric, Gas chromatography/mass spectrometry.

Correspondence to R. Timón (Fax. +34 927257 461; e-mail: rtimon@unex.es). 